\title{
Qualidade da operação de preparo do solo em três velocidades de deslocamento
}

\section{Quality of the soil preparation operation in three displacement speeds}

\author{
Antonio Alves Pinto \\ Universidade Estadual Paulista \\ E-mail: antonioufca@gmail.com \\ OrcID: https://orcid.org/0000-0001-8615-2387
}

Felipe Thomaz da Camara

Universidade Federal do Cariri

E-mail: felipe.camara@ufca.ed

OrcID: https://orcid.org/0000-0003-2853-9908

Leandro Alves Pinto

Universidade Estadual Paulista

E-mail: leandroalvespinto96@gmail.com

OrcID: https://orcid.org/0000-0003-3917-5652

Tamires Doroteo de Souza

Universidade Federal do Paraná

E-mail: tamiresdoroteo@gmail.com

OrcID: https://orcid.org/0000-0002-5593-7680

Resumo: O uso da mecanização agrícola permitiu a realização de práticas de cultivo mais eficientes nas lavouras. Objetivou-se avaliar a qualidade da operação de preparo do solo com grade aradora em três velocidades de deslocamento utilizando o Controle Estatístico de Processos (CEP). As velocidades utilizadas foram 3, 4 e $5 \mathrm{~km} \mathrm{~h}^{-1}$. O trator utilizado foi da marca New Holland, modelo 7630, com potência de $80 \mathrm{~kW}$ (108 cv) no motor, acoplado a grade aradora. Foram avaliadas a capacidade de campo operacional e o rendimento de campo efetivo como indicadores de qualidade da operação de preparo do solo. A capacidade de campo operacional obteve processo estável em todas as velocidades. Já o rendimento de campo efetivo obteve instabilidade de processo quando trabalhado com $3 \mathrm{~km} \mathrm{~h}^{-1}$. A aplicação do Controle Estatístico de Processo na operação preparo do solo permitiu identificação de pontos instáveis no rendimento de campo efetivo para a velocidade de $3 \mathrm{~km} \mathrm{~h}^{-1}$. A existência de variabilidade no rendimento de campo efetivo não compromete a estabilidade da operação de preparo do solo com grade aradora nas três velocidades. A capacidade de campo operacional e o rendimento de campo efetivo apresentam menor variabilidade na velocidade de $3 \mathrm{~km}$ $\mathrm{h}^{-1}$.

Palavras-chave: Carta de controle. Desempenho operacional. Eficiência de campo.

\begin{abstract}
The use of agricultural mechanization allowed for more efficient cultivation practices in crops. We aimed to evaluate the quality of the soil tillage operation with an offset disc harrow at three displacement speeds using the Statistical Process Control (SPC). The speeds were 3,4 and $5 \mathrm{~km} \mathrm{~h}^{-1}$. It's was used a brandNew Holland tractor, model 7630, with $80 \mathrm{~kW}$ (108 CV) engine power, coupled at leveling harrow. The operational field capacity and the effective field yield were evaluated as indicators of soil tillage operation quality. The operational field capacity acquired a stable process at all speeds. The effective field yield gained process instability when worked with $3 \mathrm{~km} \mathrm{~h}^{-1}$. The approach of Statistical Process Control in the soil preparation allowed the identification of unstable points in the effective field yield for a speed of $3 \mathrm{~km} \mathrm{~h}^{-1}$. The existence of variability in the effective field yield does not compromise the stability of the soil tillage operation with an offset disc harrow at the three speeds. The operational field capacity and the effective field yield show less variability in the speed of $3 \mathrm{~km} \mathrm{~h}^{-1}$.
\end{abstract}

Keywords: Control chart. Field efficiency. Operational performance. 


\section{Introdução}

A utilização da mecanização agrícola tornou mais eficiente diversas operações, sendo o trator a principal máquina utilizada na realização das atividades agrícolas permitindo assim a realização de práticas de cultivo mais eficientes (Martins et al., 2018). Conforme (Pinto et al., 2020) o trator é uma máquina auto propelida que permite a realização de práticas de cultivo mais eficientes, dentro do período considerado ótimo para as culturas.

As grades são um dos principais implementos utilizados, no preparo periódico do solo. Diferencia-se dos arados pela forma de preparo do solo bem como, por apresentar maior largura de trabalho real, resultando em elevada capacidade de campo operacional (Martins et al., 2018).

Ao avaliarem o desempenho operacional do preparo reduzido e do convencional, Fernandes e Gamero (2010), obtiveram capacidade de campo teórica de 1,28 ha $\mathrm{h}^{-1}$ para grade aradora. Corroborando com Cortez et al. (2011) que obtiveram diferença na capacidade de campo efetiva e capacidade de campo operacional em operações de preparo do solo com grade aradora, influenciada pela variação na velocidade de deslocamento.

Relacionando-se a capacidade de campo operacional com a efetiva, obtém-se a eficiência de campo das máquinas e implementos agrícolas, afetadas por vários fatores como o padrão de operação no campo, a declividade da área, a capacidade de campo e velocidade de deslocamento (Silveira et al., 2006). Tais fatos reforçam a necessidade de identificar os pontos instáveis que possa comprometer essas operações.

O Controle Estatístico de Processo (CEP) é uma ferrementa do Controle Estatístico de Qualidade (CEQ), que visa assegurar a qualidade e estabilidade dos processos, com ferramentas que auxiliam na detecção de padrões e falhas de execução. Em estudos relacionados com a melhoria das operações agrícolas o CEP é considerado eficaz na caracterização de variabilidade do processo (Silva et al., 2015; Paixão et al., 2017 ).

Silva et al. (2013) utilizaram o CEP para avaliar a qualidade da colheita mecanizada de feijão em dois sistemas de preparo do solo. Já Tavares et al. (2015) trabalhando com qualidade do recolhimento mecanizado do café, concluíram que aplicação do CEP na operação de recolhimento permite analisar, separadamente cada fase do processo, identificando pontos a serem melhorados, aumentando a qualidade da operação.

Considerando que o aumento da velocidade de trabalho, influencia na eficiência e na capacidade de campo operacional da operação, objetivou-se avaliar a qualidade da operação de preparo do solo com grade aradora em três velocidades de deslocamento utilizando o Controle Estatístico de Processos (CEP).

\section{Material e Métodos}

O experimento foi conduzido durante uma operação de preparo primário do solo, na área experimental do Centro de Ciências Agrárias e da Biodiversidade da Universidade Federal do Cariri (UFCA) - Crato-CE.

O trator utilizado foi da marca New Holland, modelo 7630, com potência de $80 \mathrm{~kW}$ (108 cv) no motor, acoplado a grade aradora, com duas seções contendo 16 discos recortados com 20" de diâmetro e largura de trabalho teórica de $2,1 \mathrm{~m}$.

Foram determinadas a velocidade de deslocamento, a largura de trabalho real, a capacidade de campo efetiva, capacidade de campo operacional e o rendimento de campo efetivo, porém, no trabalho apenas a capacidade de campo operacional e o rendimento de campo efetivo foram utilizadas como fatores de qualidade da operação gradagem, as demais variáveis foram utilizadas na obtenção do rendimento de campo efetivo.

A velocidade foi determinada cronometrando-se o tempo gasto para o trator operar com a grade por uma distância de 40 metros, realizando-se 10 repetições para cada velocidade e calculado a média de velocidade. A largura real foi determinada considerando-se a média de três passadas da grade, com uso de 
trena, realizando-se 30 repetições. A capacidade de campo efetiva foi determinada pelo produto da largura real e velocidade real, com os dados sendo expressos em hectares por hora, conforme a equação 1.

$$
\mathrm{CCE}=\frac{\mathrm{Lr} * \mathrm{Vr}}{10000}
$$

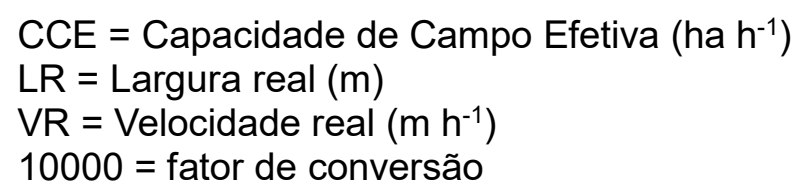

A capacidade de campo operacional foi determinada cronometrando-se o tempo gasto para preparar uma área de $1000 \mathrm{~m}^{2}$, considerando-se os tempos gastos com manobras. Para cada velocidade foi determinado coletado 30 valores de $\mathrm{CcO}$ para cada velocidade.

O rendimento de campo efetivo foi obtido pela relação entre as capacidades de campo operacional e capacidade de campo efetiva, com os dados sendo expressos em porcentagem, conforme a equação 2.

$$
\mathrm{RcE}=\left(\frac{\mathrm{CCO}}{\mathrm{CCE}}\right) * 100
$$

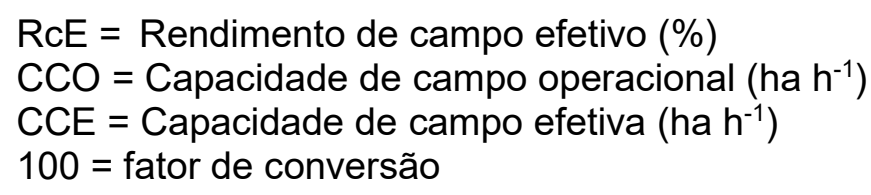

O delineamento experimental seguiu os padrões estabelecidos pelo controle estatístico de processo, nos quais os dados foram coletados ao longo do tempo (Silva et al., 2015). Foram avaliados 30 valores de capacidade de campo operacional $(\mathrm{CcO})$ e o rendimento de campo efetivo (RcE), utilizados como fatores de qualidade da operação gradagem, para cada velocidades de deslocamento $\left(3\right.$, 4 e $\left.5 \mathrm{~km} \mathrm{~h}^{-1}\right)$, totalizando 90 valores para cada variável no final do monitoramento da operação de gradagem.

Os dados foram submetidos à análise descritiva para a visualização do comportamento dos dados. Foram calculadas as medidas de tendência central (média aritmética e mediana), medidas de dispersão (amplitude, desvio-padrão e o coeficiente de variação) e medidas de assimetria e curtose. Para verificar a normalidade dos dados realizou-se o teste de Anderson-Darling (Silva et al., 2015).

Em conjunto à análise descritiva dos dados aplicou-se o Controle Estatístico de Qualidade (CEQ) para verificar a variabilidade e estabilidade do processo (Silva et al., 2015), utilizando-se ferramentas do Controle Estatístico de Processos (CEP). Foram utilizados os gráficos sequenciais (run charts) para detecção de padrões de comportamento (agrupamento, mistura, tendência e oscilação) e cartas de controle de valores individuais e amplitude móvel, empregando como parâmetros de qualidade a capacidade de campo operacional e o rendimento de campo efetivo, visando identificar causas de variabilidade não inerentes ao processo, consideradas críticas. Os limites de controle foram calculados considerando a médias mais ou menos três vezes o desvio-padrão das variáveis. Todas as análises estatísticas foram realizadas no programa estatístico Minitab ${ }^{\circledR}$

\section{Resultados e Discussão}

Observou-se que nas três velocidades avaliadas, houve proximidade entre os valores de média e mediana, para a capacidade de campo operacional $(\mathrm{CcO})$, com os valores de amplitude e desvio-padrão baixos, fator que indica uma tendencia de normalidade dos dados (Tabela 1).

Os coeficientes de variação para $\mathrm{CcO}$ em cada velocidades apresentaram valores baixos $(<10)$, de acordo com a classificação de Gomes (2009), indicando baixa variabilidade dos dados. Fato este considarado bom, tendo em vista que na literatura os coeficientes de variação da $\mathrm{CcO}$ variam de baixo $(<10)$ a muito alto (>30), (Carvalho Filho et al ., 2013; Cunha et al., 2016; Martins et al., 2018). 
Tabela 1. Estatística descritiva para a capacidade de campo operacional ( $\mathrm{CcO})$ e redimento de campo efeteivo (RcE).

\begin{tabular}{|c|c|c|c|}
\hline & \multicolumn{3}{|c|}{$\mathrm{CcO}\left(\mathrm{ha} \mathrm{h}^{-1}\right)$} \\
\hline & $3 \mathrm{~km} \mathrm{~h}^{-1}$ & $4 \mathrm{~km} \mathrm{~h}^{-1}$ & $5 \mathrm{~km} \mathrm{~h}^{-1}$ \\
\hline Média & 0,54 & 0,73 & 0,89 \\
\hline Mediana & 0,54 & 0,74 & 0,90 \\
\hline Amplitude & 0,06 & 0,12 & 0,14 \\
\hline DP & 0,02 & 0,03 & 0,03 \\
\hline CV & 3,32 & 4,38 & 3,90 \\
\hline $\mathrm{Ck}$ & -0.91 & $-0,62$ & $-0,57$ \\
\hline Cs & 0,05 & 0,40 & 0.06 \\
\hline \multirow[t]{3}{*}{ Nomalidade } & $0,538^{N}$ & $0,397^{\mathrm{N}}$ & $0,238^{N}$ \\
\hline & \multicolumn{3}{|c|}{$\operatorname{RcE}(\%)$} \\
\hline & $3 \mathrm{~km} \mathrm{~h}^{-1}$ & $4 \mathrm{~km} \mathrm{~h}^{-1}$ & $5 \mathrm{~km} \mathrm{~h}^{-1}$ \\
\hline Média & 92,79 & 92,32 & 92,54 \\
\hline Mediana & 92,96 & 92,72 & 92,59 \\
\hline Amplitude & 7,93 & 12,17 & 11,01 \\
\hline DP & 2,19 & 3,08 & 2,65 \\
\hline CV & 2,36 & 3,34 & 2,87 \\
\hline Ck & -0.36 & $-0,31$ & $-0,31$ \\
\hline Cs & -0.36 & $-0,54$ & -0.01 \\
\hline Nomalidade & $0,237^{N}$ & $0,341^{\mathrm{N}}$ & $0,097^{N}$ \\
\hline
\end{tabular}

DP- Desvio-padão; CV- Coeficiente de variação; Ck - Coeficiente de curtose; Cs - Coeficiente de assimetria; N: distribuição normal.

Os coeficientes de curtose obtiveram dispersões negativas dos dados no $\mathrm{CcO}$ de todas as velocidades de deslocamento, indicando que a distribuições são platicúrticas, ou seja, achatamento da curva na região central. Para os coeficientes de assimetria (Cs) do $\mathrm{CcO}$ os valores foram todos positivos indicando afastamento dos valores da variável para a direita (assimetria a direita) em relação ao valor central, sendo que para a velocidade de deslocamento de 3 e $5 \mathrm{~km} \mathrm{~h}^{-1}$ o coeficiente de assimetria é classificado com pequeno $(|<0,15|)$, enquanto na velocidade $4 \mathrm{~km} \mathrm{~h}^{-1}$ o $\mathrm{Cs}$ foi moderado entre $(\mid 0,15$ a $1,0 \mid)$, conforme descrito por Tavares et al. (2015).

Para o redimento de campo efeteivo (RcE) na Tabela 1, observou-se que nas três velocidades, ocorreu proximidade entre os valores de média e mediana, com os valores de amplitude, desvio-padrão e coeficientes de variação baixos. Já os valores de Cs foram negativos indicando assimetria à esquerda. Ressalta-se que todos os Cs obtiveram assimetria de pequena a moderada (Tavares et al., 2015), conforme o teste de Anderson-Darling, todas as velocidades obtiveram distribuição normal em todas as variáveis.

Avaliando os resultados sintetizados dos gráficos sequenciais (Tabela 2) observou-se que na CcO houve formação de padrões de oscilação nas velocidades de deslocamento de 3 e $4 \mathrm{~km} \mathrm{~h}^{-1}$, indicando não aleatoriedade dos dados nessas velocidades, indicando que as variáveis não estão sujeitas somente à ação de causas naturais de variação no processo. Conforme Silva et al. (2015), os padrões de oscilação indicam a ocorrência de causas especiais de variação ao longo do tempo.

Tabela 2. Valores-padrão de probabilidades dos gráficos sequenciais para a capacidade de campo operacional $(\mathrm{CcO})$ e redimento de campo efeteivo $(\mathrm{RcE})$.

\begin{tabular}{|c|c|c|c|}
\hline \multirow{2}{*}{ Padrões } & \multicolumn{3}{|c|}{$\mathrm{CcO}\left(\mathrm{ha} \mathrm{h}^{-1}\right)$} \\
\hline & $3 \mathrm{~km} \mathrm{~h}^{-1}$ & $4 \mathrm{~km} \mathrm{~h}^{-1}$ & $5 \mathrm{~km} \mathrm{~h}^{-1}$ \\
\hline Agrupamento & $0,947^{\text {ns }}$ & $0,963^{\text {ns }}$ & $0,843^{\text {ns }}$ \\
\hline Mistura & $0,063^{\text {ns }}$ & $0,067^{\text {ns }}$ & $0,157^{\mathrm{ns}}$ \\
\hline Tendência & $1,000^{\text {ns }}$ & $1,000^{\text {ns }}$ & $0,383^{\text {ns }}$ \\
\hline Oscilação & $0,000^{*}$ & $0,000^{*}$ & $0,617^{\mathrm{ns}}$ \\
\hline \multirow{2}{*}{ Padrões } & \multicolumn{3}{|c|}{ RcE (\%) } \\
\hline & $3 \mathrm{~km} \mathrm{~h}^{-1}$ & $4 \mathrm{~km} \mathrm{~h}^{-1}$ & $5 \mathrm{~km} \mathrm{~h}^{-1}$ \\
\hline Agrupamento & $0,000^{*}$ & $0,068^{\text {ns }}$ & $0,069^{\text {ns }}$ \\
\hline Mistura & $1,000^{\text {ns }}$ & $0,931^{\text {ns }}$ & $0,933^{\text {ns }}$ \\
\hline Tendência & $0,006^{*}$ & $0,379^{\text {ns }}$ & $0,383^{\text {ns }}$ \\
\hline Oscilação & $0,994^{\mathrm{ns}}$ & $0,617^{\mathrm{ns}}$ & $0,616^{\mathrm{ns}}$ \\
\hline
\end{tabular}

ns Não significativo p>0,05 (aleatoridade dos dados); * - Significativo $p<0,05$ (não aleatoridade dos dados). 
No RcE observou-se que apenas na velocidade de $3 \mathrm{~km} \mathrm{~h}^{-1}$ apresentou padrões de agrupamento e tendência, conforme Silva et al. (2015), a ocorrência desses padrões indica que os dados podem estar próximos de sair do controle, ou seja os valores podem estar próximos de ficarem acima ou abaixo dos padrões desejados para a variável analisada.

Observa-se na carta controle de valores individuais e amplitude móvel, que o processo está sob controle para a $\mathrm{CcO}$, em todas as velocidades de deslocamento, com todos os valores dentro dos limites inferiores e superiores, foi observado variação dos valores abaixo e a acima da média $(\bar{X})$ em todas as velocidades de deslocamento (Figura 1).

No RcE (Figura 2) observou-se que na velocidade de $3 \mathrm{~km} \mathrm{~h}^{-1}$, houve a ocorrência de 4 pontos fora de controle, sendo um acima do limite superior (UCL) e três a baixo do limite inferior de controle (LCL), tal fato provavelmente foi em função de falhas de medida na determinação da largura real, uma vez que os pontos abaixo do limite de controle ocorreram nos seis primeiros pontos amostrais, coincidindo com o período de adaptação com a coleta dos pontos, não sendo observado nas demais velocidades em que a mão-de-obra já estava treinada.

Em processos agrícolas devido à alta variabilidade existente, os pontos fora de controle presente na carta são provenientes de fatores extrínsecos ao processo (Silva et al., 2013), não sendo suficiente para comprometer a qualidade da operação tendo em vista que os valores de RcE estão próximos ou acima de $90 \%$ valores considerados ótimos em operações de preparo de solo (Balastreire,1987).
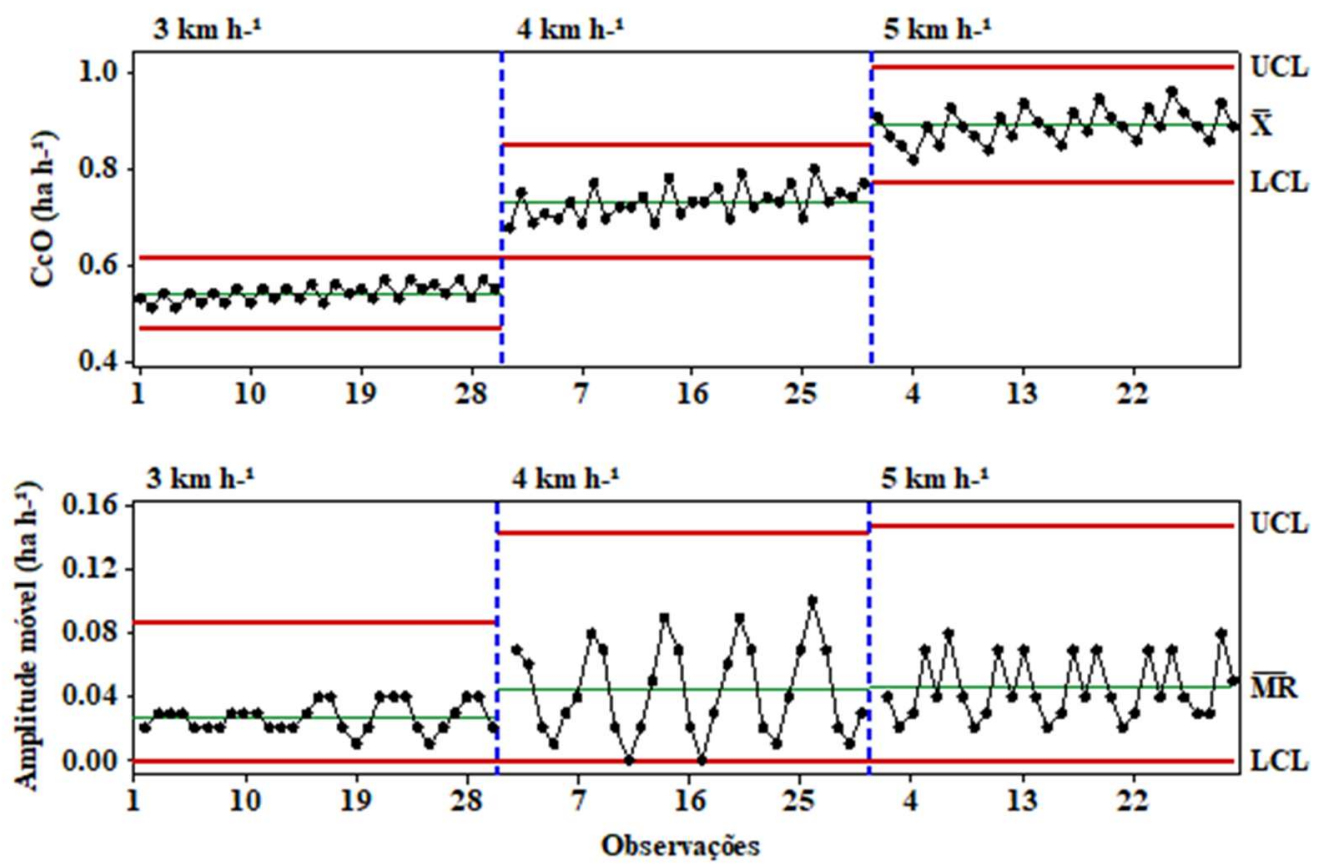

Figura 1. A. Carta de controle de valores individuais e amplitude móvel para capacide de campo operacional $(\mathrm{CcO})$.

Nas velocidades de 4 e $5 \mathrm{~km} \mathrm{~h}^{-1}$ não foram observados pontos fora de controle, porém é possível verificar alta variabilidade do processo nas duas velocidades, proveniente provavelmente de falhas na determinação (medida) da variável, uma vez que, as medições tendem a serem realizadas mais rápidas com o aumento da velocidade de trabalho, além disso, também pode ser devido a falhas proveniente da mão-deobra (operador), tendo em vista que, para realizar manobras o operador precisa reduzir a velocidade, variando assim o tempo gasto com cada manobra resultando em variação na $\mathrm{CcO}$ e das variáveis dependentes dela como o RcE . Conforme Paixão et al. (2017), os fatores extrínsecos ao processo (causas especiais) estão relacionados aos 6 M's (Matéria-prima, Medida, Máquina, Mão-de-obra, Método e Meio ambiente). 

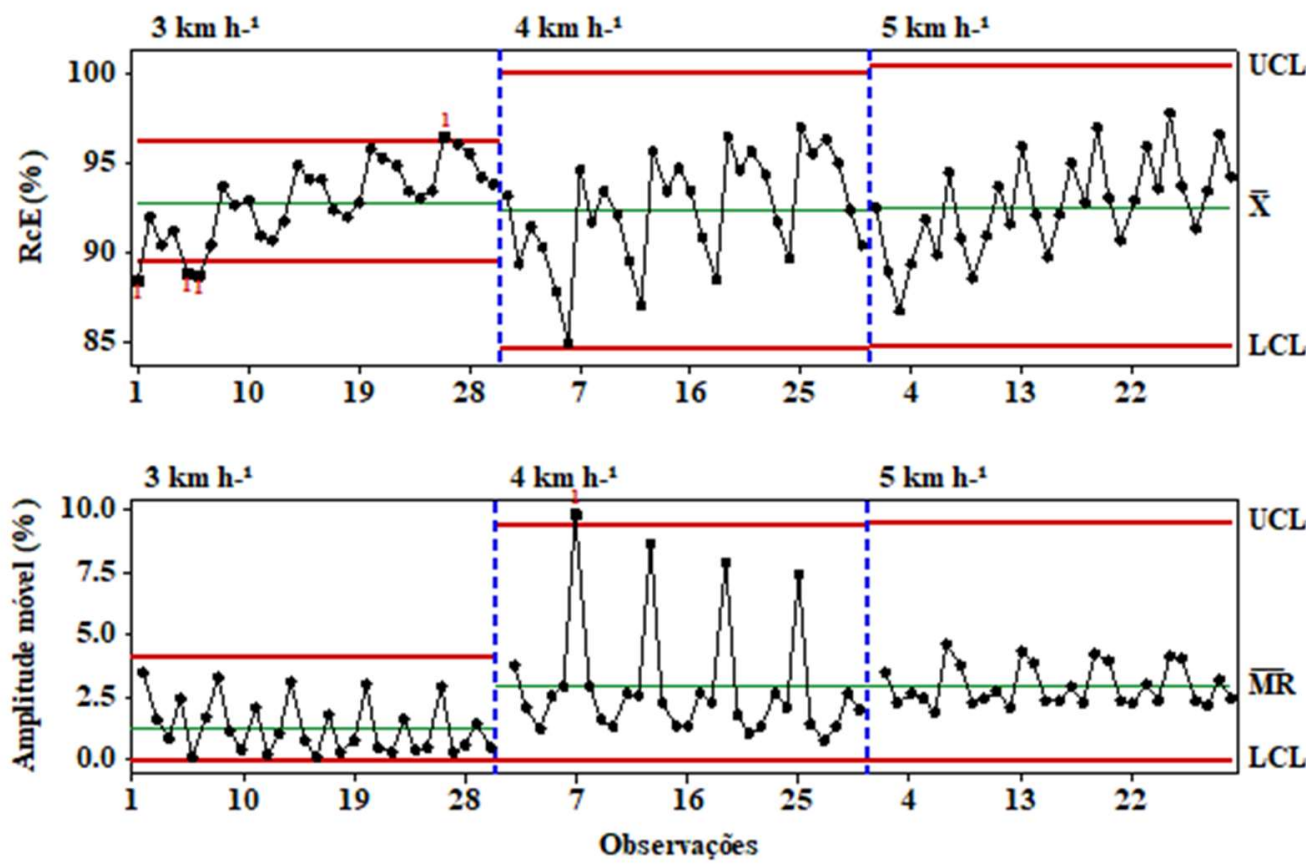

Figura 2. Carta de controle de valores individuais e amplitude móvel para o rendimento de campo efetivo (RcE).

\section{Conclusões}

A aplicação do Controle Estatístico de Processo na operação de preparo do solo permitiu identificação de pontos instáveis no rendimento de campo efetivo para a velocidade de $3 \mathrm{~km} \mathrm{~h}^{-1}$.

A existência de variabilidade no rendimento de campo efetivo não compromete a estabilidade da operação de preparo do solo com grade aradora nas três velocidades.

A capacidade de campo operacional e o rendimento de campo efetivo apresentam menor variabilidade na velocidade de $3 \mathrm{~km} \mathrm{~h}^{-1}$

\section{Referências}

Balastreire, L.A. (1987) Máquinas agrícolas. São Paulo: Manole, 307p

Carvalho Filho, C.A., Cortez, J.W., Santos, V.M.L., Arcoverde, S.N., Nagahama, H.J. (2013). Desempenho do trator agrícola em função das marchas e das proporçõe de biodiesel. Energia na Agricultura, 28(3), 135142. https://doi.org/10.17224/EnergAgric.2013v28n3p135-142.

Cortez, J.W., Ferreira, B.J.M., Alves, A.D.S., Moura, M.R.D., Nagahama, H.J. (2011) Desempenho operacional do conjunto trator-implementos nas operações de preparo do solo. Nucleus, 8(2), 177-184.

Cunha, J.P.B., Silva, F.M., Dias, R.E.B.A. (2016) Eficiência de campo em diferentes operações mecanizadas na cafeicultura. Coffee Science, 11(1), 76-86. http://www.sbicafe.ufv.br:80/handle/123456789/8175

Fernandes, J.C., Gamero, C.A. (2010). Avaliação do desempenho das máquinas agrícolas na implantação da cultura do girassol. Energia na Agricultura, 25(2), 74-87.https://doi.org/10.17224/EnergAgric.2010v25n2p74-87 Gomes, F.P. (2009). Curso de estatística experimental. 15.ed. Piracicaba: FEALQ, 451p.

Martins, M.B., Sandi, J., Souza, F.L.; Santos, R.S.; Lanças, K.P. Otimização energética de um trator agrícola utilizando normas técnicas em operações de gradagem. Engenharia na agricultura, v.26, n.1, p. 52-57, 2018. https://doi.org/10.13083/reveng.v26i1.852 
Paixão, C.S.S., Alcântara, A.S., Voltarelli, M.A., Tavares, T.O., Silva, R.P. (2017). Monitoring of soybean mechanical harvesting as a function of plot shapes. Revista Engenharia Agrícola, 37(4), 689-698. http://dx.doi.org/10.1590/1809-4430-eng.agric.v37n4p689-698/2017

Pinto, A.A., Pinto, L.A., Camara, F.T. (2020). Comprimento do talhão na operação de preparo do solo com grade média. Agrarian, 13(49), 419-425. https://doi.org/10.30612/agrarian.v13i49.10915.

Silva, R.P., Voltarelli, M.A., Cassia, M.T. (2015). Controle de qualidade em operações agrícolas mecanizadas. 1 ed. Jaboticabal: SBEA, 244p.

Silva, R.P., Cassia, M.T., Voltarelli, M.A., Compagnon, A.M., Urlani, C.E.A. (2013). Qualidade da colheita mecanizada de feijão (Phaseolus vulgaris) em dois sistemas de preparo do solo. Revista Ciência Agronômica, 44, 61-69. http://dx.doi.org/10.1590/S1806-66902013000100008

Silveira, G.M., Yanai, K., Kurachi, S.A.H. (2006). Determinação da eficiência de campo de conjuntos de máquinas convencionais de preparo do solo, semeadura e cultivo. Revista Brasileira de Engenharia Agrícola e Ambiental, 10(1), 220-224. http://dx.doi.org/10.1590/S1415-43662006000100032

Tavares, T.O., Santinato, F., Silva, R.P., Voltarelli, M.A., Paixão, C.S.S., Santinato, R. (2015). Qualidade do recolhimento mecanizado do café. Coffee Science, $10(4), \quad 455-463$. http://www.sbicafe.ufv.br:80/handle/123456789/8148 